\title{
Helicobacter pylori eradication induce platelet recovery in idiopathic thrombocytopenic purpura
}

\author{
Blood 2001; 97: 812-4
}

\author{
G. Emilia, G. Longo, M. Luppi, G. Gandini, M. Morselli, L. Ferrara, S. Amarri, K. Cagossi, G. Torelli \\ Dipartimento di Scienze Mediche, Sezione di Medicina Interna e Ematologia, Università degli Studi di Modena
}

\begin{abstract}
SUMMARY
Recent reports have suggested an association between Helicobacter pylori infection and idiopathic thrombocytopenic purpura (1TP). The prevalence of H pylori infection and the effect of its eradication in a series of 30 ITP patients were investigated. H pylori infection has been documented in 13 patients (43.33\%) by ${ }^{13} \mathrm{C}$ urea breath test and confirmed by histologic examination. Bacterium eradication with antibiotics, obtained in 12 of 13 infected patients $(92.3 \%)$, led to a complete response in 4 (33.33\%) and to a partial response (platelets $\left.90 \times \mathrm{IO}^{\wedge} \mathrm{L}-120 \times \mathrm{IO} \mathrm{V}^{\prime} \mathrm{L}\right)$ in 2 (16.66\%). The response was maintained for a median of 8.33 months, but 1 patient relapsed 7 months after eradication. Search for $H$ pylori infection seems appropriate in ITP patients at diagnosis. Bacterium eradication provides a new good option for a non immunosuppressive treatment in some ITP patients. (Blood 2001; 97: 812-814)
\end{abstract}

\begin{abstract}
a tutti noto il ruolo dell'Elicobacter Pilori (EP) nel causare gastriti e ulcera peptica, nonché adenocarcinoma gastrico e linfomi delle mucose. Più recentemente l'infezione da EP è stata coinvolta nella patogenesi di molte altre affezioni che non riguardano direttamente l'apparato gastroenterico ma che vanno prese in considerazione perché alcune sono anche di competenza reumatologica. A tale proposito ci è sembrato interessante segnalare in questa rubrica l'articolo sopra citato il quale rappresenta una messa a punto di altri lavori o segnalazioni che rilevavano l'alta frequenza di infezioni da EP in pazienti con piastrinopenia idiopatica che rispondevano al trattamento eradicante dell'infezione.

L'importanza del lavoro, ove i risultati fossero confermati in casistiche più ampie, consiste prevalentemente in due aspetti; il primo concerne l'eviden-
\end{abstract}

za di una associazione statisticamente significativa tra piastrinopenia idiopatica e infezione da EP, la seconda è data dalla possibilità di curare questa affezione con la semplice terapia antibiotica e gastroprotettiva piuttosto che con cortisonici, IgEV o immunosoppressori. La dimostrazione inoltre che l'infezione possa determinare la comparsa di patologia immunitarie, anche di competenza reumatologica, apre un campo di ricerca che riguarda il rapporto, mai completamente chiarito, tra infezioni e patologie autoimmuni incluse quelle di competenza reumatologica. È probabile che in futuro ricerche più approfondite in questo campo possano contribuire a migliorare le nostre conoscenze sui meccanismi eziopatogenetici di altre malattie reumatiche.

Mauro Galeazzi 